\title{
Note sur une épizootie de mélioïdose porcine au Cambodge
}

\author{
SREY THONN, E. LEBON. SAPHON, et R. TRIAU
}

Un intérêt grandissant s'attache depuis plusieurs années à la mélioïdose dont le domaine géographique s'est brusquement agrandi depuis la guerre du Pacifique. De son foyer originel dans l'Asie des moussons, la maladie a gagné certaines zones tropicales d'Amérique et d'Australie où elle s'est manifestée sous la forme épizootique (ovins, caprins, porcins).

D'autre part nos connaissances sur le germe spécifique (Malleomyces ou Whitmorella pseudomallei), sur l'épidémiologie ef sur la clinique de la maladie, sur les procédés de diagnostic et sur les moyens thérapeutiques se trouvent enrichies par un grand nombre de travaux récents auxquels ont participé les Instituts Pasteur d'Indochine.

La mise au point la plus complète et la plus récente nous paraît être la monographie de FOURNIER et CHAMBON (1), auteurs dont la contribution personnelle de recherches est considérable.

Dans son foyer originel sud-asiatique, la mélioïdose s'étaił jusqu'à présent manifestée sous la forme de cas sparadiques (avec, parfois, quelques cas groupés). Mais, depuis la fin de la guerre, l'importation de bêtes de races sélectionnées, qui est une des formes de l'aide économique étrangère, semble devoir modifier la situation épizootologique. En même temps, et à cette occasion, l'action accrue de nos moyens d'investigation se montre susceptible de découvrir de nouveaux aspects.

C'est pourquoi nous croyons intéressant de relater une épizootie porcine de mélioìdose, la première qui ait été observée au Cambodge.

Reçu pour publication : avril 1960.

Rev. Elev. Méd. vét. Pays trop., 1960, 13, no 2-3.
Dans un élevage de porcs provenant d'Australie et du Japon - élevage du Service vétérinaire national installé à une dizaine de kilomètres de Phnom-Penh - de magnifiques truies Yorkshire pesant de 200 à $280 \mathrm{~kg}$ se mettent à avorter. Peu de symptômes ensuite si ce n'est une légère parésie du train postérieur accompagnée d'une petite poussée fébrile irrégulière. Et pourtant clles meurent dix à quinze jours après leur avortement.

Sur les cadavres on prélève des os longs : la médulloculture est négative.

Un jour, une truie « record »-280 kg meurt brusquement. Cette fois on nous adresse quelques-uns de ses viscères: rate, foie, poumons. lis présentent de nombreux nodules caséeux. L'ensemencement du caséum donne rapidement des cultures de bacille de Whitmore à l'état pur.

Appelés à la station, nous examinons frois autres truies malades. Etat général excellent, mais on note une légère faiblesse du train arrière et une discrète hyperthermie. Une hémoculture met en évidence, à nouveau, la présence de bacille de Whitmore dans le sang d'une des malades.

La morlalité continue dans cet élevage et, en l'espace d'un mois, nous recevons six nouveaux cadavres ( 5 truies, 1 verrat).

A l'autopsie on observe les lésions suivantes: poumons infiltrés, plèvres purulentes, ulcères des organes digestifs : estomac, intestin, caecum, valvule iléo-caecale. On retrouve de petits abcès miliaires ou des nodules caséeux disséminés dans les poumons, le foie, le mesentère, les ganglions, la rate.

Fait curieux : les cultures obtenues à partir des nodules caséeux des cinq truies de cette sèrıe ne font apparaître que des pasteurelles, salmo- 
nelles et pyocyaniques. Pas de bacille de Whitmore malgré l'emploi du milieu de Mac Conkey recommandé par FOURNIER ef CHAMBON.

Chez le verrat, par contre, en plus des lésions déjà décrites, on remarque une hypertrophie testiculaire considérable. A la coupe, les deux glandes se montrent farcies de micro-abcès caséeux, desquels on isole Malleomyces pseudamallei à l'état pur.

Là encore, les tentatives de culture à partir des autres organes atteints : poumons, ganglions, foie, ne permettent pas de mettre en évidence le bacille de Whitmore. La médulloculture reste négative.

Peu après la maladie est observée dans deux autres stations du Service vétérinaire.

L'une se trouve dans la Province de Takéo à $100 \mathrm{~km}$ au sud-est de Phnom-Penh. On y isole deux nouvelle souches : la première par culture de pus d'un nodule splénique où elle se trouve associée à des streptocoques. La deuxième souche, isolée par médulloculture chez un porcelet atteinf d'orchite, est accompagnée de pyocyaniques.

Le dernier centre d'élevage où nous avons pu observer la mélioĩdose est situé dans la Province de Pursat 'à 200 km au nord de Phnom-Penh. Mêmes faits cliniquès: avortement chez les truies, parésie du train postérieur, mort en dix à quinze jours. On nous adresse le poumon, le foie et la rate d'un verrat mort en dix jours. Dans les nodules pulmonaires nous isolons du bacille de Whitmore associé à des staphylocoques ef colibacilles.

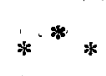

Cette épizootie de mélioïdose porcine apparue brusquement et atteignant en premier lieu des animaux importés pose la question de l'origine du contage.

Est-ce une mélioĩdose importée ? ou s'agit-il de cas autochtones? La maladie existe-t-elle parmi les porcs du Cambodge depuis longtemps, non diagnostiquée, méconnue?

Cette dernière hypothèse nous paraît la plus probable pour les raisons suivantes:

$10 \mathrm{Si}$ quelques animaux atteints ont été achetés en Australie, pays où la mélioïdose est bien connue, du moins au Queensland, par contre un autre lot de porcs, également atteint, a été importé du Japon où l'affection n'a jamais été signalée (*). Enfin certains sujets nés au Cambodge n'ont pas été épargnés.

$2^{\circ}$ L'importation a eu lieu plus d'un an avant l'apparition des premiers cas de mélioïdose. Les porcs sont arrivés au Cambodge alors qu'ils étaient fort jeunes (10 mois) et qu'ils ne pesaient qu'une cinquantaine de kilos. Ils se sont parfaitement développés, sont devenus d'excellents reproducteurs et leur embonpoint faisait plaisir à voir jusqu'au jour de leur maladie.

3o Si on reconnait des porteurs sains de bacille de Whitmore, - «formes asymptomatiques 》 où le germe n'est mis en évidence que par la recherche systématique sur carcasse de boucherie - par contre on n'a pas encore signalé le cas d'excréteurs de bacilles.

En admettant que parmi les animaux introduits au Cambodge il se trouvait des porteurs sains, l'affection n'aurait pas pris cette allure retardée, meurtrière, frappant principalement les animaux imporfés.

On sait que les porcs de race perfectionnée sont beaucoup plus sensibles aux maladies que les races locales, très rustiques. Ces dernières se montreraient peu sensibles à la mélioïdose alors que les porcs d'élevages iaméliorés la contracteraient plus aisément.

40 Autre argument épidémiologique, le Cambodge se trouve au milieu de la zone endémoenzootique la plus ancienne et la plus importante de la mélioïdose. L'existence au Cambodge de la mélioïdose chez l'homme a été signalée par GAMBIER (2) en 1930:

En 1953, notre collègue le $D^{r}$ vétérinaire HUARD eut l'occasion d'observer une épizootie - qui sévissait parmi les chevaux des voitures de place de Phnom-Penh. A cette occasion il isola. d'abcès caséeux viscéraux un germe que de LAJUDIE à I'Institut Pasteur de Saïgon identifia comme étant le bacille de Whitmore.

L'existence de la mélioìdose porcine au Cambodge devait nécessairement passer inaperçue tant qu'il n'existait dans ce pays aucun grand élevage de porcs, ce qui fut le cas jusqu'à une époque toute récente. Les petits élevages dissé-

(*) Toutefois l'apparition de cas de mélioidose porcine au Viet-Nam parmi des bêtes récemment importées du Japon a été portée à notre connaissance : voir FOURNIER. Rapport annuel de l'Institut Pasteur du Viet-Nam, année 1958, p. 12. 
minés sur le territoire n'ont jamais fait l'objet d'investigations bactériologiques. Le paysan cambodgien qui voyait mourir son pore sous sa paillote subissait cette grosse perte sans avoir recours à qui que ce soit.

Au Viet-Nam, étot qui jouxte le Cambodge, l'existence de la mélioïdose porcine a été reconnue par NGUYEN BA LUONG (3) en 1956 avec isolement de souches. Dès 1952, FOURNIER (4) a constaté que les porcs du Viet-Nam, porcs présumés sains (amenés aux abattoirs de Saïgon), avaient dans leur sérum des agglutinines naturelles anti-M. pseudomallei; ceci dans une proportion importante ( 85 p. 100 des bêtes) et a des taux parfois fort élevés (1/160).

On peut donc considérer comme acquis que la mélioidose porcine existe depuis fort longtemps au Cambodge et que le cheptel autochtone, au prix d'une mortalité de base qui nous est inconnue, possède un certain degré d'immunité contre la maladie.

En définitive, le processus de l'épizootie que nous relatons nous paraît le suivant : des sujets sélectionnés provenant de pays indemnes de mélioidose, sujets par conséquent neufs et hautement réceptifs ont été introduits dans un pays situé en pleine zone d'enzootie. La maladie a éclaté parmi eux sous la forme épizootique et s'est étendue au cheptel local en surmontant son immunité naturelle relative, ou en frappant des bêtes qui avaient échappé à l'infection immunisante. On doit admettre aussi que le développement de nos investigations bactériologiques a dépisté des cas autochtones qui, il y a quelques années, n'quraient. pas été rattachés à leur étiologie véritable.

Enfin, l'imporiance numérique des élevages allant en augmentant, les chances de manifestations épizootiques à partir d'un fond enzootique latent se trouvent accrues.

- Il est bien difficile de préciser comment la contagion s'est effectuée. Cependant l'origine tellurique apparaît la plus vraisemblable à la lumière des travaux de VAUCEL (5) puis de FOURNIER ef CHAMBON (1).

Dans les cas présentement décrits, certains porcs sont tombés malades peu de.temps après le «bouclage ». Cette opération consiste à pas- ser un gros anneau métallique à travers la cloison nasale. Ainsi les animaux sont-ils gênés pour fouiller - et dévaster - le sol avec leur groin. La blessure provoquée par la pose de l'anneau peut constituer une excellente porte d'entrée pour les germes du sol.

La contagion inter-animale semble devoir être écartée : la maladie est apparve simultanément, vers la fin de la saison des pluies, dans trois stations d'élevages, distantes de plusieurs centaines de kilomètres les unes des autres. Les animaux y avaient été répartis peu de temps après leur importation, un an avant l'épizootie. La reproduction avait commencé et des portées avaient déjà éłé enregistrées.

Dans une même station où se trouvaient des sujets de races Berkshire, Yorkshire Large White ef des Yorkshire Middle White, seuls les Large White furent touchés.

Notons qu'avant nous d'autres auteurs (NGUYEN BA LUONG, auteurs australiens) ont écarté aussi l'hypothèse de la contagion inter-animale.

Cette occasion nous a permis de dégager quelques notions cliniques sur la mélioïdose porcine, maladie très peu décrite par les auteurs de langue française.

Symptomatologie généralement très pauvre: oscillations thermiques, diminution inconstante de l'appétit. Dans certains cas les animaux ont mangé jusqu'à leur mort. Assez fréquemment on note une discrète parésie du train postérieur, n'allant jamais jusqu'à la paralysie vraie. Ce symptôme relativement constant évoque la possibilité d'une toxine neurotrope qui serait élaborće par Malleomyce pseudomallej.

La lenteur de l'évolution est assez caractéristique : certains sujets fébriles ont survécu pendant dix à douze semaines. Dans ce cas leur peau prend une coloration particulière très pâle, couleur porcelaine.

D'une manière générale la mélioïdose porcine revêt essentiellement la forme septico-pyohémique. Les localisations suppurées, pleuro-pulmonaires, intestinales, spléniques, ganglionnaires ou testiculaires contiennent du bacille de Whitmore à l'état pur ou associé à d'autres germes.

Des poussées septicémiques peuvent se produire dont témoignent des hémocultures positives,

Les formes localisées sont assez fréquentes: 
orchite où le germe est retrouvé à l'état pur, ostéïte avec isolement du bacille par médulloculture.

Le contraste est frappant entre l'absence relative de signes' cliniques et l'existence de grosses lésions suppurées. Celles-ci cependant ne sont pas toujours présentes et dès 1936, l'observation de GIRARD (6) a attiré l'attention sur les formes inapparentes. Dans d'autres cas les bêtes dépérissent, se cachectisent et présentent parfois du jetage : l'autopsie révèle de nombreux ef gros abcès viscéraux.

Du point de vue du diagnostic clinique et biologique la maladie pose un véritable rébus. Seules des hémocultures répétées pourraient permettre, dans certains cas, l'isolement du germe et, par suite, son identification.

Le diagnostic nécropsique nous paraît plus aisé : on doit penser à la mélioïdose en présence d'abcès miliaires nodulaires ou autres dispersés dans tous les viscères.

C'est l'examen bactériologique qui donnera la clé du problème: l'isolement du bacille de Whitmore n'offre aucune difficulté particulière sauf s'il est masqué par d'autres germes. (Encore, dans ce cas, la résistance de $M$. pseudomallei a la plupart des antibiotiques autres que le chloramphénicol permet-il de surmonter cette difficulté.)

Le diagnostic bactériologique doit comporter non seulement la culture mais aussi linoculation au cobaye, ou mieux, l'inclusion d'une grosse masse de produit pathologique selon la méthode de NGUYEN VAN Al (7).

Le dépistage par la recherche de l'allergie, si décevant en médecine humaine, paraît donner de meilleurs résultats en médecine vétérinaire à condition de prolonger l'observation pendant au moins deux semaines. II semble qu'on puisse utiliser soif les mélioidines de OLDS ef LEWIS (8), soil l'anligène $K$ extrait par CHAMBON (9) qu'on adminiștre par injection dans la paupière inférieure. Cependant cette méthode de dépistage n'a guère été employée que chez les ovins et chez les caprins. Des recherches dans ce sens méritent d'être entreprises chez les porcs.

\section{RÉSUMÉ ET CONCLUSIONS}

Nous décrivons une épizootie meurtrière de mélioìdose apparue chez les porcs sélectionnés provenant de pays indemnes de mélioïdose et importés au Cambodge c'est-à-dire en pleine zone endémo-enzootique. A la suite et à l'occasion de cette épizootie plusieurs cas groupés ont été dépistés dans le cheptel autochtone.

Cette observation vient à l'appui de l'opinion de COTTEW (10) et de FOURNIER et CHAM$B O N$ (1) sur l'opportunité de travaux expérimentaux pour obtenir un vaccin efficace. L'application d'un tel vaccin aux sujets sélectionnés importés au Cambodge serait hautement désirable.

Nos observations apportent une contribution à l'étude clinique de la mélioïdose porcine. Des signes neurologiques (parésie du train postérieur) y sont pour la première fois mentionnés.

La réceptivité de l'homme à la mélioïdose et la gravité chez lui de cette infection ajoutent à l'importance économique de la question un puissant intérêt en matière de protection de la santé humaine.

Service vétérinaire national et Institut Pasteur du Cambodge.

\section{BIBLIOGRAPHIE}

1. FOURNIER (J.) et CHAMBON (L.). - la mélioïdose et le bacille de Whitmore, Ed méd. Flammarion Paris 1958.

2. GAMBIER (A.). - Bull. Soc. Path. exot. 1930, $23: 436$.

3. NGUYEN BA LUONG. - Bull. Soc. Path. exot. 1956; $49: 25$.

4. FOURNIER (J.). - Rapport sur le fonctionnement technique de l'Institut Pasteur de Saigon en 1952 (1 vol. Saigon 1953, I DE O). p. 40.

5. VAUCEL $(M$.$) . - Mélioïdose in Médecine$ tropicale, Paris, 1952-1959, Ed. méd. Flammarion, 1493-1507 G.

6. GIRARD (G.). - Bull. Soc. Path. exot. 1936, $29: 717-6$.

7. NGUYEN VAN Al. - Bull. Soc. Poth. exot. 1957, $50: 11$.

8. OLDS (R. J.) et LEWIS (F. A.). - Aust. vet. J., $1954,30: 253$.

9. CHAMBON (L.) STAUB (A. M.) et BOURDET (M. C.). - Ann. Inst. Posteur, 1957, $93: 376$.

10. COTTEIW (G. S.). - Aust, vet. J., 1955, 31 : 155. 


\section{SUMMARY}

\section{An Epizootic of Melioidosis in Pigs in Cambodge.}

An outbreak of melioidosis in pigs, with a heavy mortality is described. The animals had been imported from an area known to be free of the disease, into Cambodge which is an enzootic area. At the same time as the outbreak in these animals, several cases were noted in local animals.

This observation confirms the opinion of Cottew and of Fournier and Chambon, on the necessity for experimental work in developing an efficient vaccine.

The observations made, add to the knowledge on porcine melioidosis. Nervous symptoms (paraplegia of the hind quarters) are recorded for the first time.

The susceptibility of man to melioidosis and the grave syndrome which follows infection adds to the purely economic importance of the disease, an important public health factor.

\section{RESUMEN}

\section{Trabajo sobre una epizootia de melioidosis porcina en Camboya.}

Describimos una epizootia de melioidosis, que produjo gran número de muertes, aparecida en cerdos selectos procedentes de países indemnes a dicha enfermedad, e importados a Camboya, zona en que es endemo-enzootica. Es con ocasión de esta epizootia, cuando algunos casos han sido descubiertos en grupos autóctonos.

Esta observación viene a reforzar la opinión de Cottew y de Fournier y Chambon sobre la conveniencia de nuevas investigaciones para obtener una vacuna eficaz. La aplicación de tal vacuna a los animales seleccionados importados a Camboya sería altamente ventajosa.

Nuestras observaciones aportan unos conocimientos al estudio clínico de la melioidosis porcina. Signos neurológicos (parexia del tercio posterior) son mencionados por primera vez. La' receptividad del hombre a la melioidosis y la gravedad en él de esta infección, añaden a su importancia económica un nuevo interés en materia de sanidad pública. 